Check for updates

Cite this: RSC Adv., 2017, 7, 36722

\title{
High-performance transparent ultraviolet photodetectors based on inorganic perovskite $\mathrm{CsPbCl}_{3}$ nanocrystals
}

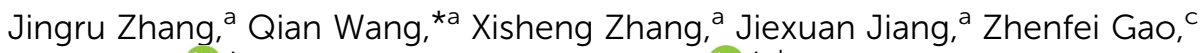 \\ Zhiwen Jin (D) *a and Shengzhong (Frank) Liu (D)*ab
}

\begin{abstract}
Inorganic lead halide perovskite nanocrystals ( $\mathrm{CsPbCl}_{3} \mathrm{NCs}$ ) with excellent ultraviolet (UV) light absorption, high carrier mobility, long carrier diffusion lengths, and long-term stability are good candidates as smart materials for transparent optoelectronic devices. In this study, transparent UV photodetectors (PDs) based on $\mathrm{CsPbCl}_{3} \mathrm{NCs}$ were fabricated for the first time. The optimized device exhibited visible light transmittance approximately $90 \%$, strong absorption of UV light in the wavelength from $300 \mathrm{~nm}$ to $410 \mathrm{~nm}$, good photoresponsivity (1.89 $\mathrm{A} \mathrm{W}^{-1}$ ), and a high on/off ratio (up to $10^{3}$ ). Meanwhile, the rise and decay response times of the device were less than $41 \mathrm{~ms}$ and $43 \mathrm{~ms}$, respectively. Furthermore, we performed detailed analysis of the effects by employing $\mathrm{CsPbCl}_{3} \mathrm{NCs}$ in assembled films and final devices using various characterization methods. The simple fabrication and remarkable UV photodetection capabilities of $\mathrm{CsPbCl}_{3} \mathrm{NCs}$ make them promising semiconducting candidates in optoelectronic applications.
\end{abstract}

Received 13th June 2017 Accepted 11th July 2017

DOI: $10.1039 / c 7 r a 06597 c$

rsc.li/rsc-advances of $\mathrm{ZnO}$ usually lasts between $30 \mathrm{~s}$ and $200 \mathrm{~s}$, because of adsorption and desorption of $\mathrm{O}_{2}$ trapping states. ${ }^{12,13}$ Synthesizing novel $\mathrm{ZnO}$-based nanocomposites by modifying the surface of $\mathrm{ZnO}$ is a promising solution. In the past decades, there have been numerous reports on the surface capping of $\mathrm{ZnO}$ using in inorganic materials, organic materials, carbon materials, and $\mathrm{Au}$ nanoparticles. However, the effect is still not ideal. ${ }^{14-17}$ In parallel, oxide semiconductors are usually n-type, making it difficult to realize transparent systems with complementary circuits. ${ }^{18}$

To develop the next generation of UV-transparent PDs, new semiconductor materials with easy fabrication, strong light absorption, high charge carrier mobility, stability in air, few defects, and bipolarity are needed. In particular, perovskites possess the merits of high charge carrier mobility and strong light absorption 10 times greater than those of organic dyes, small exciton binding energies, and long carrier diffusion lengths of up to $175 \mu \mathrm{m}$, making them promising candidates for use in such PD applications. ${ }^{19-25}$ More recently, inorganic perovskites $\mathrm{CsPbX}_{3}(\mathrm{X}=\mathrm{Cl}, \mathrm{Br}, \mathrm{I})$ have attracted research efforts as future optoelectronic materials since they are more stable in air than organic-inorganic perovskite materials. ${ }^{\mathbf{2 6 - 3 0}}$

Inorganic perovskite $\mathrm{CsPbCl}_{3}$ is a wide-bandgap semiconductor, which is transparent to visible radiation but sensitive to UV radiation. ${ }^{31}$ Such absorption, mainly limited to wavelengths shorter than $400 \mathrm{~nm}$, makes it attractive for visibleblind UV detection. In recent years, the optical bandgap of $\mathrm{CsPbCl}_{3}$ has been adjusted by reducing the crystallite size to the nanometer scale to form nanocrystals (NCs). ${ }^{32,33}$ The optical bandgap increases as the size decreases, making $\mathrm{CsPbCl}_{3} \mathrm{NCs}$ 
capable of meeting the different requirements of various fields applications. However, to the best of our knowledge, no efficient $\mathrm{UV}$ detection devices have yet been developed based on $\mathrm{CsPCl}_{3}$ NCs.

In this study, we analyzed in detail the effects of employing $\mathrm{CsPbCl}_{3}$ NCs in assembled films and final devices. Furthermore, we designed the first transparent UV PD based on inorganic $\mathrm{CsPbCl}_{3}$ NCs. The fabricated device exhibited high UV sensitivity (1.89 $\left.\mathrm{A} \mathrm{W}^{-1}\right)$, a high on-off current ratio $\left(10^{3}\right)$, short rise and decay times ( $41 \mathrm{~ms}$ and $43 \mathrm{~ms}$, respectively), and longterm photostability, which suggesting that $\mathrm{CsPbCl}_{3} \mathrm{NCs}_{\text {have }}$ great potential for use in high-performance transparent UV PDs.

\section{Results and discussion}

Solution-processing techniques have been used to fabricate the $\mathrm{CsPbCl}_{3}$ NCs. Spin-coating was used to fabricate $\mathrm{CsPbCl}_{3} \mathrm{NCs}$

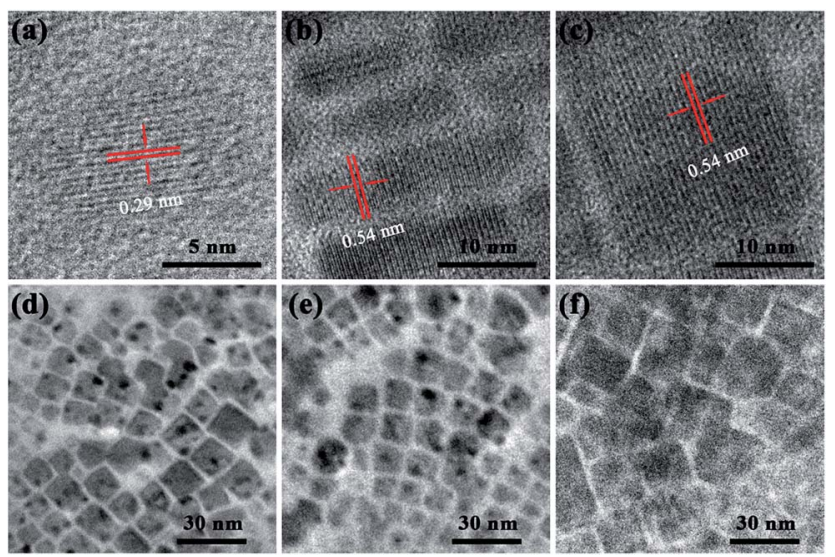

Fig. 1 (a)-(c) Typical HRTEM images of $\mathrm{CsPbCl}_{3} \mathrm{NCs}$ obtained using different reaction conditions. TEM images of cubic $\mathrm{CsPbCl}_{3} \mathrm{NCs}$ after washing (d) two, (e) four, and (f) six times. films for high-performance PD devices. First, we compared $\mathrm{CsPbCl}_{3} \mathrm{NCs}$ prepared at a low temperature $\left(90^{\circ} \mathrm{C}\right)$ in various reaction times $(5 \mathrm{~s}, 10 \mathrm{~s}$, and $20 \mathrm{~s})$. We found evidence to support the previous reports that $\mathrm{CsPbCl}_{3} \mathrm{NC}$ formation occurs through seed-mediated nucleation and that further growth occurs through self-assembly and oriented attachment. ${ }^{34}$ The typical high-resolution transmission electron microscope (HRTEM) images presented in Fig. 1a-c reveal the fine crystalline structures of the $\mathrm{CsPbCl}_{3}$ NCs, indicating assemblies of quantum dot (QD), platelet, and cubic $\mathrm{CsPbCl}_{3} \mathrm{NCs}$, respectively, with dimensions of about 5-20 nm.

It is found that monodispersed spherical $\mathrm{CsPbCl}_{3}$ quantum dots (QDs) are well-separated and uniform in size with diameter $\sim 5 \mathrm{~nm}$. This is confirmed by transmission electron microscope (TEM), as shown in Fig. 1a. The high-resolution TEM (HR-TEM) imaging reveals that the QDs are highly crystalline in structure. As shown in Fig. 1a, the lattice fringes of the $\mathrm{CsPbCl}_{3}$ QDs have a spacing of $c a .0 .31 \mathrm{~nm}$, well in agreement with the interplane spacing of the cubic-phase $\mathrm{CsPbBr}_{3}$ for the (111) crystal planes. ${ }^{35}$ As the reaction time rises from $5 \mathrm{~s}$ to $10 \mathrm{~s}$, colloidal $\mathrm{CsPbCl}_{3}$ platelet was formed with obvious morphology shown in Fig. 1b. Careful measurements indicate that the $\mathrm{CsPbCl}_{3}$ nanocubes are $c a .10 \mathrm{~nm}$ in length. The HR-TEM image reveals that the lattice fringe of a represent cubic is $0.54 \mathrm{~nm}$, in consistent with the (001) plane of cubic crystal structure $\mathrm{CsPbCl}_{3} \cdot{ }^{35}$ By further enhanced the reaction times $(20 \mathrm{~s})$, the $\mathrm{CsPbCl}_{3}$ cubics were synthesized. As presented in Fig. 1c. The HR-TEM image (Fig. 2f) highlights the $\mathrm{CsPbCl}_{3}$ cubics with a well-defined crystalline structure with a cubic lattice parameter of $0.54 \mathrm{~nm}$, along with the crystalline direction of (001). ${ }^{35}$

It is well known that the NC quality (purity and solubility) is important to fabricate large-area, crack-free, low-roughness perovskite thin films. Here, we demonstrated a highly efficient purification process involving using a hexane/methyl acetate (MeOAc) mixed solvent to control the surface ligand density on
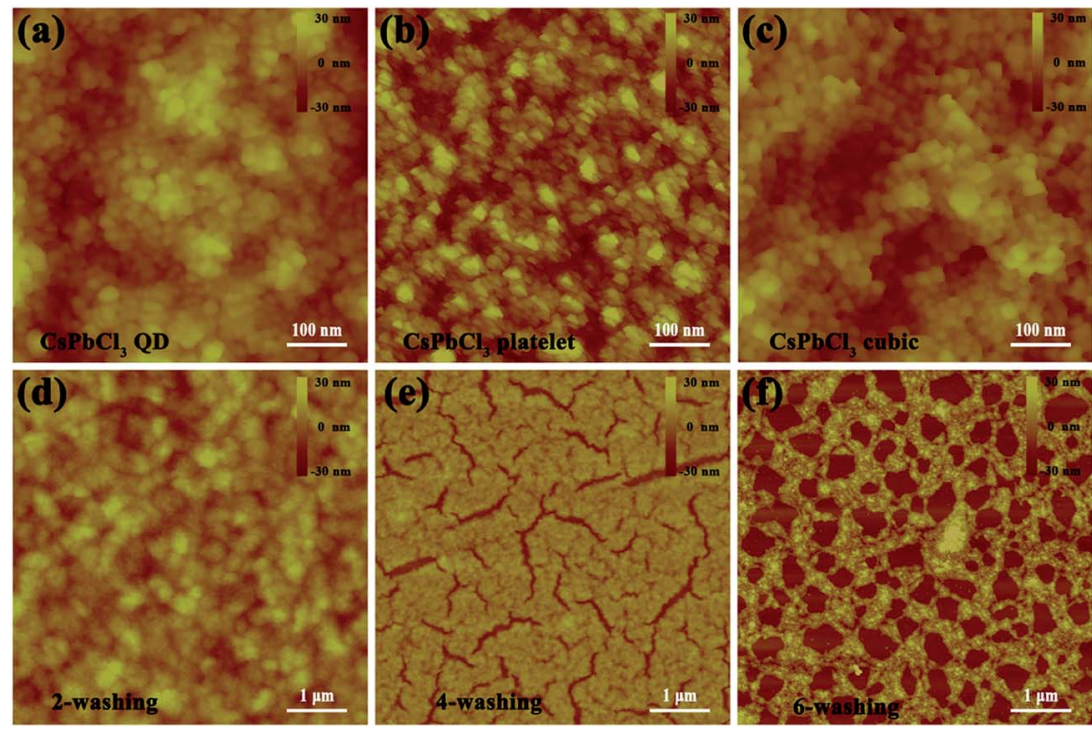

Fig. 2 AFM images of the films fabricated using $\mathrm{CsPbCl}_{3}$ in (a) $\mathrm{QD}$, (b) platelet, and (c) cubic form. AFM images of the film fabricated using cubic $\mathrm{CsPbCl}_{3}$ after washing (d) two, (e) four, and (f) six times. 
(a)

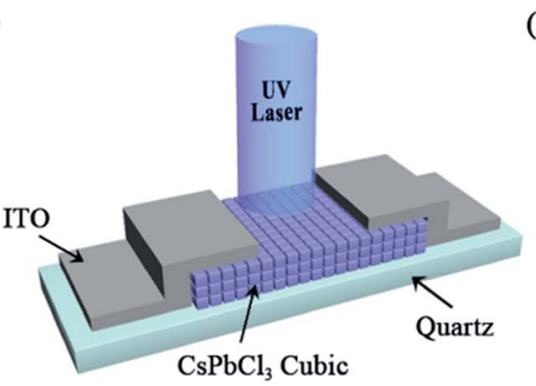

(d)

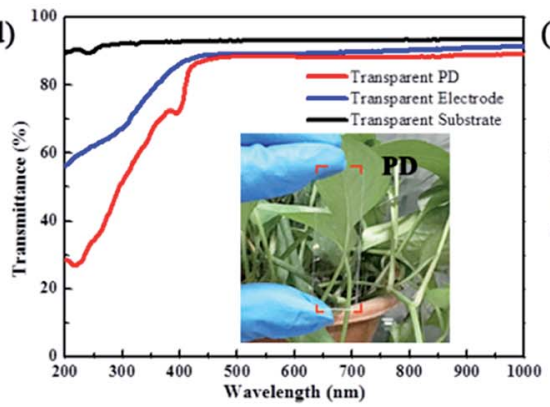

(b)

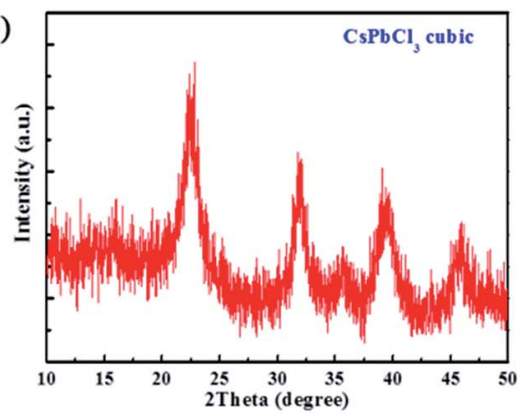

(e)

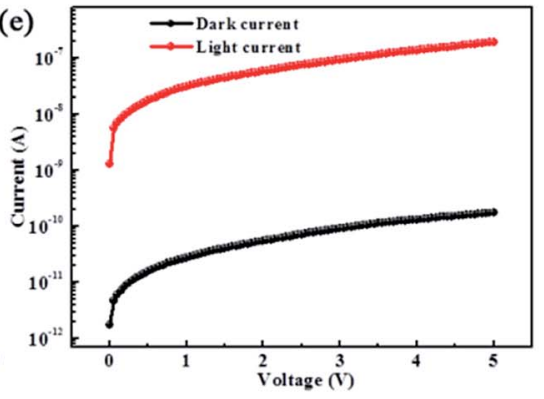

(c)
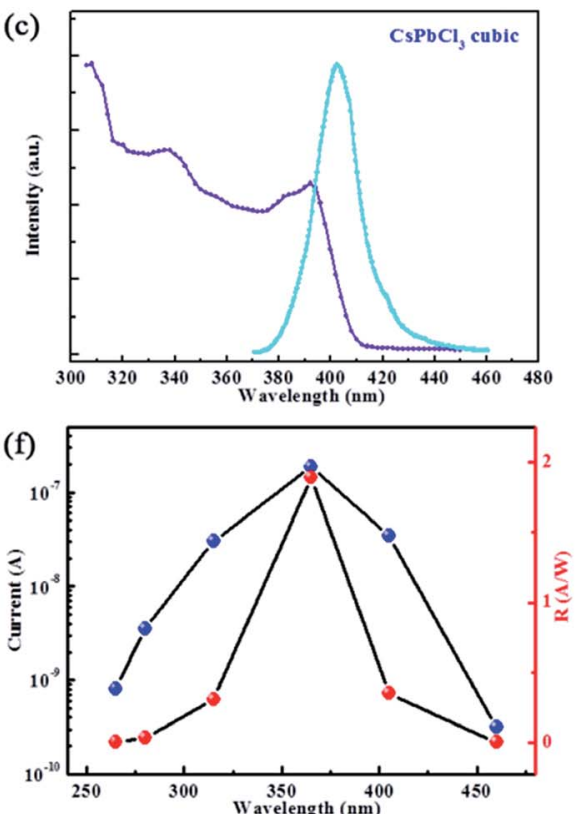

Fig. 3 (a) Schematic of the device. (b) XRD pattern and (c) absorption and emission spectra of cubic $\mathrm{CsPbCl}_{3}$. (d) Transmittance spectra of the bare substrate, electrode, and PD device. (e) $I-V$ logarithm curves of the PD device under $365 \mathrm{~nm}$ illumination (10 $\mathrm{mW} \mathrm{cm}^{-2}$ ) and in darkness. (f) Photocurrent spectrum and $R$ spectrum of the fabricated PD.

the $\mathrm{CsPbCl}_{3}$ NCs to realize the high purity and solubility. The transmission electron microscopy (TEM) images that obtained after washing the cubic $\mathrm{CsPbCl}_{3} \mathrm{NCs}$ two, four, and six times are exhibited in Fig. 1d-f, respectively. The grain corners gradually become blurred as the number of washings increases. Thus, excessive purification resulted in obvious aggregation and precipitation due to the serious loss of surface ligands. Based on these results, it was decided to wash the $\mathrm{CsPbCl}_{3} \mathrm{NCs}$ only two times for purification. ${ }^{36}$

In order to investigate the microstructure and morphology of the $\mathrm{CsPbCl}_{3}$ thin films, atomic force microscope (AFM) measurements were performed. Fig. 2a-c present AFM images of the individual films based on QD, platelet, and cubic $\mathrm{CsPCl}_{3}$ $\mathrm{NCs}$, respectively, and clearly reveal the small $\mathrm{CsPbCl}_{3}$ grains. Fig. 2d-f display AFM images of the films fabricated with $\mathrm{CsPbCl}_{3}$ cubic purified at different times (two, four, and six times, respectively). The film used the $\mathrm{CsPbCl}_{3}$ cubic that was washed twice is clearly smooth and uniform. However, the film became cracked after the cubic washing four times. Furthermore, washing six times caused a large number of holes to form in the cubic $\mathrm{CsPbCl}_{3}$ film, which would extend the carrier transport length. These results also indicate that the film quality is the best after washing twice. ${ }^{37}$

The cubic $\mathrm{CsPbCl}_{3}$ NC-based PD is illustrated schematically in Fig. 3a. The device contains a typical ITO/ $\mathrm{CsPbCl}_{3} \mathrm{NCs} /$ quartz stack: the $\mathrm{CsPbCl}_{3} \mathrm{NC}$ film was fabricated by spin-coating approach with $100 \mathrm{~nm}$ thickness; afterwards, a pair of interdigitated ITO electrodes (100 nm thick) was thermally evaporated onto the films through a shadow mask, resulting in a channel width/length ratio of $200 \mu \mathrm{m} / 5 \mu \mathrm{m}$. Fig. 3b shows a typical X-ray diffraction (XRD) pattern of cubic $\mathrm{CsPbCl}_{3} \mathrm{NCs}$.
Apparently, the $\mathrm{CsPbCl}_{3}$ crystallizes into a cubic structure (Pm3m space group) with no observable impurity phases. ${ }^{38}$ The absorption and photoluminescence (PL) spectra were measured and are presented in Fig. 3c. The $\mathrm{CsPbCl}_{3} \mathrm{NCs}$ exhibit strong absorption from $300 \mathrm{~nm}$ to $410 \mathrm{~nm}$ and a narrow band-edge emission peak at $402 \mathrm{~nm}$, which is consistent with the literature. ${ }^{39,40}$ The absorption implies that PDs based on $\mathrm{CsPbCl}_{3} \mathrm{NCs}$ will intrinsically be visible-blind.

The PD fabricated using cubic $\mathrm{CsPbCl}_{3}$ NCs shows approximately $90 \%$ optical transmittance in the visible light region. For comparison, the transmittance spectra of the bare substrate and transparent electrode are also collected in Fig. 3d. The worse transmittances of the transparent electrode and $\mathrm{CsPCl}_{3}$ $\mathrm{PD}$ in the UV region, especially for the $\mathrm{CsPbCl}_{3} \mathrm{PD}$, are attributable to strong absorption in the UV region. The photograph inserted in Fig. $3 d$ confirms that the PD has high transparency. Note that the green plants behind the transparent device can easily be seen. Fig. 3e provides the dark and light $I-V$ curves of the $\mathrm{CsPbCl}_{3} \mathrm{PD}$, where the light curve was obtained under $365 \mathrm{~nm}$ illumination $\left(10 \mathrm{~mW} \mathrm{~cm}^{-2}\right)$. The device exhibits a high signal-to-noise ratio of about $10^{3}$, implying that the PD is highly sensitive, which may be attributable to the high lightabsorption efficiency of the $\mathrm{CsPbCl}_{3} \mathrm{NCs}^{41}$

The $\mathrm{CsPbCl}_{3} \mathrm{PD}$ also exhibits high spectral selectivity (photocurrent and responsivity $(R)$ ), which can clearly be seen in Fig. 3f. The $R$ is given by the following equation: $:^{42}$

$$
R=\frac{I_{\text {light }}-I_{\text {dark }}}{P_{\text {ill }}}
$$

where $I_{\text {light }}$ is the current density under illumination, $I_{\text {dark }}$ is the dark current density, and $P_{\text {ill }}$ is the power density of the incident illumination. It is evident that the photocurrent increases above 



Fig. 4 (a) On-off switching properties measured under $365 \mathrm{~nm}$ light illumination $\left(10 \mathrm{~mW} \mathrm{~cm}{ }^{-2}\right)$ at a bias of $5 \mathrm{~V}$. (b) Time-resolved photocurrent of the fabricated PD. (c) On-off cycling test results measured under $365 \mathrm{~nm}$ light illumination (10 $\left.\mathrm{mW} \mathrm{cm}^{-2}\right)$ at a bias of $5 \mathrm{~V}$. (d) Fit curve for photocurrent versus incident optical power. (e) Absorption spectra of $\mathrm{CsPbCl}_{3}$ in QD, platelet, and cubic form. (f) On-off switching properties of the PDs based on $\mathrm{CsPbCl}_{3}$ in QD, platelet, and cubic form.

the bandgap energy of the $\mathrm{CsPbCl}_{3} \mathrm{NCs}(3.1 \mathrm{eV}$ at a wavelength of $400 \mathrm{~nm})$, confirming the intrinsic visible-blindness of the $\mathrm{CsPbCl}_{3} \mathrm{PD}$. When the wavelength is $450 \mathrm{~nm}$, the photocurrent is very low since the incident light energy is less than the bandgap of the $\mathrm{CsPbCl}_{3}$ NCs. However, when the wavelength is shorter than $365 \mathrm{~nm}$, the photocurrent decreases gradually with decreasing wavelength, which is mainly due to the different external quantum efficiencies of the materials, as previously reported. ${ }^{43}$ The PD exhibits the strongest response to $365 \mathrm{~nm}$ light. Therefore, $365 \mathrm{~nm}$ light was employed to assess the performance of the $\mathrm{CsPbCl}_{3} \mathrm{PD}$.

The time-dependent photoresponse of the PD measured by periodically turning it on and off in $5 \mathrm{~s}$ intervals is presented in Fig. 4a. The photocurrent rapidly increases to a stable peak value $\left(1.89 \times 10^{-7} \mathrm{~A}\right)$ upon the application of $365 \mathrm{~nm}$ illumination and then quickly decreases to its initial value $\left(1.71 \times 10^{-10} \mathrm{~A}\right)$ upon the light being turned off, revealing that the $\mathrm{CsPbCl}_{3} \mathrm{PD}$ has highly stable and reproducible characteristics. It should be note that the rise time and decay time of the PDs are defined as the time taken for the initial current to increase or decrease to $90 \%$ of the peak value, respectively. ${ }^{44}$ The rise and decay times of the present device are $41 \mathrm{~ms}$ and $43 \mathrm{~ms}$ (Fig. 4b), respectively, indicating that the $\mathrm{CsPbCl}_{3} \mathrm{PD}$ has ultra-fast responses compared to those the traditional transparent ZnO-based UV PDs, whose typical response speeds have been reported to be tens or hundreds of seconds (seen in Table 1 ). ${ }^{4-52}$ The on/off cycle testing results are depicted in Fig. 4c. After 1000 repeated on/off cycles, the on current $\left(1.83 \times 10^{-7} \mathrm{~A}\right)$ and off current $(1.81 \times$ $10^{-10}$ A) do not suffer any appreciable deterioration, and the 
Table 1 Comparison of the characteristic parameters of photodetectors

\begin{tabular}{lllll}
\hline Films & $\begin{array}{l}\text { Rise time } \\
(\mathrm{s})\end{array}$ & $\begin{array}{l}\text { Decay time } \\
(\mathrm{s})\end{array}$ & $\begin{array}{l}R \\
\left(\mathrm{~A} \mathrm{~W}^{-1}\right)\end{array}$ & Reference \\
\hline $\mathrm{CsPbCl}_{3} \mathrm{NC}$ & 0.041 & 0.043 & 1.89 & This work \\
$\mathrm{ZnO} \mathrm{NC}$ & 0.1 & 1 & 61 & 45 \\
$\mathrm{ZnO}_{\mathrm{SnO}}$ nanofibers & 32.2 & 7.8 & - & 46 \\
$\mathrm{LaAlO}_{3}$ single crystal & - & - & 0.0718 & 47 \\
$\mathrm{SrTiO}_{3}$ single crystal & - & - & 0.213 & 48 \\
$\mathrm{SrTiO}_{3}$ single crystal & - & - & 0.03 & 49 \\
$\mathrm{GaN} \mathrm{film}_{\mathrm{NiO} \mathrm{film}}$ & 0.28 & 0.45 & 0.34 & 50 \\
$\mathrm{GdNiO}_{3}$ & 0.266 & 0.2 & 4.5 & 51 \\
& - & - & 0.23 & 52 \\
\hline
\end{tabular}

device maintains a high on/off ratio above $10^{3}$. These parameters are beneficial for practical applications.

Fig. $4 \mathrm{~d}$ shows the photocurrent and photoresponsivity $(R)$ as a function of the light intensity. The photocurrent increases as the illumination intensity increases, which is as expected since the number of photogenerated carriers is proportional to the absorbed photon flux. The fit line reveals that the photocurrent is proportional to the light intensity. In this study, the highest value of $R$ was found to be $1.89 \mathrm{~A} \mathrm{~W}^{-1}$ upon device optimization. Compared with other traditional transparent materials based UV PDs (seen in Table 1), ${ }^{45-52}$ the remarkably photo-response performance by the $\mathrm{CsPbCl}_{3}$ PDs is attributed to its larger crystal size, which are favorable for carrier mobility, leading to enhanced photoresponse.

The absorbing properties of the QD, platelet, and cubic $\mathrm{CsPbCl}_{3}$ PDs as determined using UV-vis spectroscopy are presented in Fig. 4e. To the best of our knowledge, the optical bandgap increases as the NC size decreases. ${ }^{53,54}$ Therefore, the optical bandgap can be tuned by adjusting the reaction conditions in the $\mathrm{CsPbCl}_{3} \mathrm{NC}$ synthesis process at $90{ }^{\circ} \mathrm{C}$ using various reaction temperatures. ${ }^{55}$ The optical absorption spectra of all of the PDs (Fig. 4e) show intense absorption in the UV region. Evidently, the optical bandgap of the $\mathrm{CsPbCl}_{3} \mathrm{NCs}$ is redshifted as the crystal size increases (from QD, to platelet, to cubic), which is consistent with other reports. ${ }^{56}$

The $I-t$ curves of the QD, platelet, and cubic $\mathrm{CsPbCl}_{3}$ PDs are presented in Fig. $4 \mathrm{f}$ for comparison. All of the PDs exhibit high current responses to the UV light and excellent on-off switching performances. The distinction among the three types of $\mathrm{CsPbCl}_{3}$ NCs is that the peak current intensity increases as the crystal size increases, since the carrier mobility increases simultaneously. Large particles will reduce the number of the interface defects, resulting in higher carrier mobility and photocurrent. ${ }^{57}$

\section{Conclusions}

In conclusion, we performed detailed analysis of the effects of employing $\mathrm{CsPbCl}_{3} \mathrm{NCs}$ in assembled films and final devices using various characterization methods and finally successfully fabricated a high-performance transparent UV PD from solutionprocessed inorganic perovskite $\mathrm{CsPbCl}_{3}$ NCs. The strong UV absorption and high visible light transmittance indicate that the $\mathrm{CsPbCl}_{3}$-based UV PD is intrinsically visible-blind. As a result, the device exhibits good responsivity $\left(1.89 \mathrm{~A} \mathrm{~W}^{-1}\right)$, a high on/off ratio $\left(10^{3}\right)$, and stable properties after 1000 repeated on/off cycles. Furthermore, the rise and decay times of the present device are $41 \mathrm{~ms}$ and $43 \mathrm{~ms}$, respectively, indicating that $\mathrm{CsPbCl}_{3}$ PDs possess ultra-fast responses compared with the traditional transparent UV PDs. Finally, our investigation of the influence of using different sizes of $\mathrm{CsPCl}_{3}$ crystals in UV PDs revealed that cubic $\mathrm{CsPbCl}_{3}$ crystals yield the highest photocurrent intensity due to the increase in carrier mobility with increasing size. All of these results demonstrate that inorganic perovskite $\mathrm{CsPbCl}_{3} \mathrm{NCs}$ are among the most effective semiconductors for use in highperformance transparent UV PDs.

\section{Experimental section}

\section{Materials preparation}

Synthesis of $\mathrm{CsPbCl}_{3}$ nanocrystals: $\mathrm{Cs}_{2} \mathrm{CO}_{3}(0.415 \mathrm{~g})$, 1-octadecene (ODE, $18 \mathrm{ml})$, and oleic acid $(\mathrm{OA}, 1.76 \mathrm{ml})$ were added to a round-bottom flask and stirred under a vacuum for $30 \mathrm{~min}$ at $120^{\circ} \mathrm{C}$ as Cs-oleate. $\mathrm{PbCl}_{2}(0.25 \mathrm{~g}), \mathrm{ODE}(10 \mathrm{ml}), \mathrm{OA}(1.6 \mathrm{ml})$, and Oleylamine $(\mathrm{OAm}, 1.6 \mathrm{ml})$ were stirred in another round-bottom flask and degassed under a vacuum at $90^{\circ} \mathrm{C}$ for $1 \mathrm{~h}$ before filling the flask with $\mathrm{N}_{2}$. Then, the Cs-oleate $(0.8 \mathrm{ml})$ was swiftly injected into the reaction mixture at $90{ }^{\circ} \mathrm{C}$. The reaction was quenched by immediate immersion of the flask into an ice bath for different times. The synthesized $\mathrm{CsPbCl}_{3} \mathrm{NCs}$ (QD, platelet, and cubic) were precipitated by adding $200 \mathrm{ml}$ MeOAc. This process was repeated twice, and the $\mathrm{CsPbCl}_{3} \mathrm{NCs}$ were finally dispersed in hexane with a concentration of $50 \mathrm{mg} \mathrm{ml}^{-1}$.

\section{Device fabrication}

The PD fabrication process was as follows: the $\mathrm{CsPbCl}_{3}$ layer was fabricated by spin-coating a solution of the relevant $\mathrm{CsPCl}_{3}$ NCs (QD, platelet, or cubic) onto a cleaned quartz substrate at $2500 \mathrm{rpm}$ for $15 \mathrm{~s}$. To form the high-mobility and low-trap density $\mathrm{CsPbCl}_{3}$ layer, the prepared film was quickly dipped into a neat MeOAc solution and then a saturated $\mathrm{Pb}(\mathrm{OAc})_{2}$ MeOAc solution. This process was repeated four times, producing $\mathrm{CsPbCl}_{3}$ films with thicknesses of about $100 \mathrm{~nm}$. All of the spin-coating steps were performed in ambient air. Finally, a pair of interdigitated ITO electrodes (100 nm thick) was thermally evaporated onto the films through a shadow mask, resulting in a channel width/length ratio of $200 \mu \mathrm{m} / 5 \mu \mathrm{m}$.

\section{Characterization}

All of the electrical characteristics were recorded with an Agilent B2902A in ambient air. The monochromatic light was from a Newport Oriel $200^{\mathrm{TM}}$. Prior to the utilization of the light, the spectral and the light intensity were calibrated using a monosilicon detector. The TEM images were taken by a JEM-2100 JEOL TEM. The surface morphology was characterized by using an AFM (Dimension ICON). The thickness of the films was determined using a Profilometer. UV-vis spectra were recorded using a JASCO V-570 spectrophotometer. The samples 
were excited by a laser (Omni- $\lambda 300)$, with a wavelength of $365 \mathrm{~nm}$. The mechanical optical choppers are used to control the laser on/off. The phase compositions of the samples were characterized by a DX-2700 XRD using $\mathrm{Cu} \mathrm{K} \alpha$ radiation $(\lambda=$ $0.15418 \mathrm{~nm}$ ) in a $2 \theta$ range of $10-50^{\circ}$.

\section{Conflict of interest}

The authors declare no competing financial interests.

\section{Acknowledgements}

This work was supported by the National Nature Science Foundation of China (61674098), the China Postdoctoral Science Foundation (2016M602759 and 2017M613052), the National Key Research Program of China (2016YFA0202403 and 2017YFA0204800), the 111 Project (B14041), the National University Research Fund (GK201703026), the Innovative Research Team (IRT_14R33) and the Chinese National 1000talent-plan program (Grant No. 111001034).

\section{References}

1 J. F. Wager, Transparent Electronics, Springer, US, 2008.

2 N. Nasiri, et al., Nanoscale, 2017, 9, 2059-2067.

3 G. A. Salvatore, et al., Nat. Commun., 2014, 5, 2982.

4 J. Sun, et al., Adv. Funct. Mater., 2011, 21, 29-45.

5 X. Zhang, et al., Nanoscale, 2017, 9, 6278-6285.

6 P. Hu, et al., Nano Lett., 2013, 13, 1649-1654.

7 J. Wang, et al., Nanoscale, 2014, 6, 10734-10739.

8 Z. Jin, et al., Sci. Rep., 2014, 4, 4268.

9 M. Patel, et al., Adv. Electron. Mater., 2015, 1, 1500232.

10 B. Sun and H. Sirringhaus, Nano Lett., 2005, 5, 2408-2413.

$11 \mathrm{~K}$. Ellmer and A. Klein, ZnO and Its Applications, Springer Berlin Heidelberg, 2008.

12 J. Lu, et al., Nanoscale, 2015, 7, 3396-3403.

13 D. Gedamu, et al., Adv. Mater., 2014, 26, 1541-1550.

14 Y. K. Mishra, et al., ACS Appl. Mater. Interfaces, 2015, 7, 14303-14316.

15 A. Bera and D. Basak, ACS Appl. Mater. Interfaces, 2009, 1, 2066-2070.

16 D.-Y. Guo, et al., Sci. Rep., 2014, 4, 7469.

17 K. Liu, et al., J. Phys. Chem. C, 2010, 114, 19835-19839.

18 Z. Qi, et al., Adv. Electron. Mater., 2015, 1, 1500173.
19 J. Jiang, et al., J. Mater. Chem. A, 2017, 5, 9514-9522.

20 D. Yang, et al., Adv. Mater., 2016, 28, 5206-5213.

21 G. Maculan, et al., J. Phys. Chem. Lett., 2015, 6, 3781-3786.

22 V. Adinolfi, et al., Adv. Mater., 2016, 28, 7264-7268.

23 G. E. Eperon, et al., Energy Environ. Sci., 2014, 7, 982-988.

24 S. D. Stranks and H. J. Snaith, Nat. Nano, 2015, 10, 391-402.

25 D. Bi, et al., Sci. Adv., 2016, 2, e1501170.

26 J. Burschka, et al., Nature, 2013, 499, 316-319.

27 H. Zhang, et al., Adv. Funct. Mater., 2017, 27, 1604382.

28 Q. Wang, et al., ACS Energy Lett., 2017, 2, 1479-1486.

29 J. Liang, et al., J. Am. Chem. Soc., 2016, 138, 15829-15832.

30 A. Swarnkar, et al., Science, 2016, 354, 92-95.

31 X. Li, et al., Small, 2017, 13, 1603996.

32 X. Li, et al., Adv. Funct. Mater., 2016, 26, 2435-2445.

33 W. Liu, et al., J. Am. Chem. Soc., 2016, 138, 14954-14961.

34 T. Udayabhaskararao, et al., Chem. Mater., 2017, 29, 13021308.

35 S. Sun, et al., ACS Nano, 2016, 10, 3648-3657.

$36 \mathrm{~J} . \mathrm{Li}$, et al., Adv. Mater., 2017, 29, 1603885.

37 X. Li, et al., Adv. Funct. Mater., 2016, 26, 5903-5912.

38 N. S. Makarov, et al., Nano Lett., 2016, 16, 2349.

39 D. Amgar, et al., Nano Lett., 2017, 17, 1007-1013.

40 G. Nedelcu, et al., Nano Lett., 2015, 15, 5635-5640.

41 Z. Jin, et al., ACS Appl. Mater. Interfaces, 2016, 8, 3304333050.

42 L. Dou, et al., Nat. Commun., 2014, 5, 5404.

43 Y. Liu, et al., Adv. Mater., 2016, 28, 9204-9209.

44 Z. Jin and J. Wang, J. Mater. Chem. C, 2014, 2, 1966-1970.

45 Y. Jin, et al., Nano Lett., 2008, 8, 1649-1653.

46 W. Tian, et al., Adv. Mater., 2013, 25, 4625-4630.

47 J. Xing, et al., Opt. Lett., 2009, 34, 1675.

48 E. J. Guo, et al., Appl. Opt., 2010, 49, 2557-2560.

49 J. Xing, et al., Opt. Lett., 2007, 32, 2526-2528.

50 A. Gundimeda, et al., Appl. Phys. Lett., 2017, 110, 103507.

51 A. A. Ahmed, et al., Sens. Actuators, A, 2017, 262, 78-86.

52 L. Wang, et al., Appl. Phys. Lett., 2017, 110, 043504.

53 Z. Jin, et al., Adv. Funct. Mater., 2016, 26, 5284-5289.

54 L. Protesescu, et al., Nano Lett., 2015, 15, 3692-3696.

55 Z. Jin, et al., Sci. Rep., 2016, 6, 37106.

56 Z. Liang, et al., ACS Appl. Mater. Interfaces, 2016, 8, 2882428830.

57 Z. Jin, et al., Adv. Mater., 2016, 28, 3697-3702. 